\title{
An Improved Method to an Impulsive and Delayed Discretized Model
}

\author{
Yujing Liu, Lijun Zhang, Shujing Gao \\ Key Laboratory of Jiangxi Province for Numerical Simulation and Emulation Techniques, Gannan Normal \\ University, Ganzhou, China \\ Email: gaosjmath@126.com
}

Received 23 December 2015; accepted 25 January 2016; published 28 January 2016

Copyright (C) 2016 by authors and Scientific Research Publishing Inc.

This work is licensed under the Creative Commons Attribution International License (CC BY).

http://creativecommons.org/licenses/by/4.0/

(c) ()

\begin{abstract}
In this paper, a discretized SIR model with pulse vaccination and time delay is proposed. We introduce two thresholds $R^{*}$ and $R_{*}$, and further prove that the disease-free periodic solution is globally attractive if $R^{*}$ is less than unit and the disease can invade if $R *$ is larger than unit. The numerical simulations not only illustrate the validity of our main results, but also exhibit bifurcation phenomenon. Our result shows that decreasing infection rate can put off the disease outbreak and reduce the number of infected individuals.
\end{abstract}

\section{Keywords}

Discrete Epidemic Model, Time Delay, Pulse Vaccination, Extinction

\section{Introduction}

Infectious diseases have a great influence on the human life and socio-economy, which lead many scientists to implement more effective measures and preparedness programs. Pulse vaccination strategy (PVS) is the one of important methods to control disease, such as hepatitis B, parotitis and encephalitis B. From the theoretical results we can know that the PVS can be distinguished from the conventional strategies in leading to disease eradication at relatively low values of vaccination [1]. And one investigates under what conditions given agent can invade partially vaccinated population, i.e., how large a fraction of the population do we have to keep vaccinated in order to prevent the agent from establishing. Then a number of epidemic models in ecology can be formulated as dynamical systems of differential equation with pulse vaccination [2]-[6], of which the SIR infectious disease model is an important biologic model.

A model for the spread of an infectious disease (involving only susceptible and infective individuals) transmitted by a vector after an incubation time was proposed by Cook [7]. This is called the phenomena of time 
delay which has very important biologic meaning in epidemic models. But for the system, many authors don’t put to use the distributed delay. Because the distributed delay allows infectivity to be a function of the duration since infection up to some maximum duration. Comparing with the time be a fixed time, the distributed delay is more appropriate form and more realistic. Beretta and Takeuchi [3] did study the following continuous SIR model with distributed delay, without considering the pulse vaccination strategy:

$$
\left\{\begin{array}{l}
\frac{\mathrm{d} s(t)}{\mathrm{d} t}=b-\beta s(t) \int_{0}^{h} f(\tau) i(t-\tau) \mathrm{d} \tau-\mu_{1} s(t), \\
\frac{\mathrm{d} i(t)}{\mathrm{d} t}=\beta s(t) \int_{0}^{h} f(\tau) i(t-\tau) \mathrm{d} \tau-\left(\mu_{2}+\lambda\right) i(t), \\
\frac{\mathrm{d} r(t)}{\mathrm{d} t}=\lambda i(t)-\mu_{3} r(t),
\end{array}\right.
$$

where the infectiousness is assumed to vary over time from the initial time of infection until a duration $h$ has passed and the function means the fraction of vector population in which the time taken to become infectious is $t$. For simplicity, they let $f(t)$ be nonnegative and continuous on $[0, h]$ and assume that $\int_{0}^{h} f(\tau) \mathrm{d} \tau=1$.

In the decade years, many authors have directly studied the delay SIR epidemic models with time delays and pulse vaccination [8]-[11]. In 2010, Yanke Du and his co-workers [9] have studied an SIR epidemic model with nonlinear incidence rate and pulse vaccination:

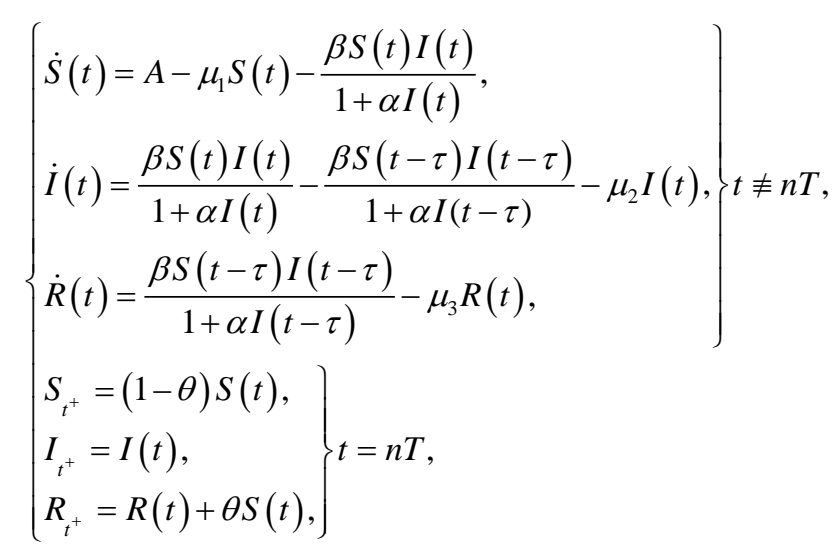

where all coefficients are positive constants. A represents the recruitment rate assuming all newborns to be susceptible. $\mu_{1}, \mu_{2}$, and $\mu_{3}$ represent the death rates of susceptible, infectious, and recovered, respectively. $\theta \quad(0<\theta<1)$ is the proportion of those vaccinated successfully, which is called impulsive vaccination rate. $T>0$ is the period of pulsing. Considering the nonlinear incidence rate $\beta S(t) I(t) /(1+\alpha I(t))$, they have found the basic reproduction $R_{0}$ and obtain an infection-free periodic solution $(S(t), 0)$ for the system. More importantly, they certified that if $R_{0}<1$, it is globally attractive, and if $R_{0}>1$, the system is permanent. But they did not study the the distributed delay. This is partly because the system is with nonlinear incidence rate and pulse vaccination, then investigation of global behavior for with the effect of saturation incidence and distributed time delay on the SIR epidemic model with a pulse vaccination is challenging.

The incidence rate plays an important role in the epidemic models. In many epidemic models, the Bilinear incidence $\beta S I$ is based on the law of mass action. This contact law is more appropriate for communicable diseases such as influenza, but not for sexually transmitted diseases. For standard incidence $\beta S I / N$, it may be a good approximation if the number of available partners is large enough and everybody could not make more contacts than practically feasible. In [12], Capasso and Serio introduced a saturated incidence rate

$\beta S I /(1+\alpha I)$ into epidemic models after studying the cholera epidemic spread in Bari in 1973, where $\beta I$ measures the infection force of the disease and $1 /(1+\alpha I)$ measures the inhibition effect from the behavioral change of the susceptible individuals when their number increases. That is to say,the saturated incidence rate tends to a saturation level when $I$ gets large. Comparing with bilinear and standard incidence, saturation incidence may be more suitable for our real world. 
On the other hand, numerical simulation is usually used to assess all kinds of continuous models and check our theoretical results. But, the statistical data of epidemic is collected and reported in discrete time, such as daily, weekly, monthly or yearly. Sometimes, they may fail generating oscillations, bifurcations, chaos and false steady states [13]. In order to be more in line with the actual, many authors are hoping to discuss discretized models, which always exhibit richer and more complicated dynamical behaviors than continuous models. For example, Masaki and Emiko [14] have used the nonstandard finite difference scheme to study the dynamics of a discretized SIR epidemic model with pulse vaccination and time delay:

$$
\left\{\begin{array}{l}
\frac{S_{n+1}-S_{n}}{h}=\Lambda-\mu_{1} S_{n+1}-\beta S_{n+1} I_{n-\omega}, \\
\frac{I_{n+1}-I_{n}}{h}=\beta S_{n+1} I_{n-\omega}-\left(\mu_{2}+\gamma\right) I_{n+1}, \\
\frac{R_{n+1}-R_{n}}{h}=\gamma I_{n+1}-\mu_{3} R_{n+1}, \\
S_{n^{+}}=(1-\theta) S_{n}, \\
I_{n^{+}}=I_{n}, \\
R_{n^{+}}=R_{n}+\theta S_{n},
\end{array}\right\} n=k \tau,
$$

where $S_{n}, I_{n}$ and $R_{n}(n=0,1,2, \cdots)$ are susceptible, infective and recovered with permanent immunity classes at $n$th step individually. $h>0$ is a positive step size. The constant $\Lambda>0$ represents the immigration rate, assuming all newborns to be susceptible. $r$ is the recovery rate. Note that the delay $\omega$ and the period of pulsing $\tau$ are positive integers, and the parameter $0<\theta<1$ is the proportion of those vaccinated successfully.

To prevent these classes of numerical instabilities, as one of numerical schemes, the nonstandard finitedifference scheme, developed by Mickens [15]-[17], has been applied to various problems in science [18]-[21]. By using this kind of scheme [16], it leads to asymptotic dynamics and numerical results are always qualitatively the same as the corresponding solutions of several ordinary differential equations for any positive step size. More importantly, This scheme has brought the creation of new numerical schemes that preserve the properties of the continuous model [2] [3] [6] [8] [10] [13] [22].

Motivated by the work of [3] [9] [14], in this paper, we are considered with the effect of saturation incidence and distributed time delay on the dynamics of a discrete SIR epidemic model with pulse vaccination:

$$
\left\{\begin{array}{l}
\frac{S_{n+1}-S_{n}}{h}=\Lambda-\mu_{1} S_{n+1}-\frac{\beta S_{n+1} \sum_{i=0}^{\omega} p_{i} I_{n-i}}{1+\alpha S_{n+1}}, \\
\frac{I_{n+1}-I_{n}}{h}=\frac{\beta S_{n+1} \sum_{i=0}^{\omega} p_{i} I_{n-i}}{1+\alpha S_{n+1}}-\left(\mu_{2}+\gamma\right) I_{n+1} \\
\frac{R_{n+1}-R_{n}}{h}=\gamma I_{n+1}-\mu_{3} R_{n+1}, \\
S_{n^{+}}=(1-\theta) S_{n}, \\
I_{n^{+}}=I_{n}, \\
R_{n^{+}}=R_{n}+\theta S_{n},
\end{array}\right\} n \in(k \tau,(k+1) \tau],
$$

where all coefficients are positive constants. $S_{n}, I_{n}$ and $R_{n}(n=0,1,2, \cdots)$ are also susceptible, infective and recovered with permanent immunity classes at $n$th step individually. $\omega>0$ is a constant integer, and $\omega h$ is the infected period, $p_{i}(i=0,1, \cdots, \omega)$ are weighting coefficients and $\sum_{i=0}^{\omega} p_{i}=1$. The notations of other parameters are the same as system (3). 
In this paper, the structure of the layout is as follows. In the next section, we mainly obtain the positivity and boundedness of the solution of the system. Furthermore, we give some important conclusions so as to make matting for the Section 3. In Section 3, we analyzed the existence and global behavior of the infection-free periodic solution of the system. The permanence of our model is discussed in Section 4. Our results are the same to Theorems 1 and 2 in system (2). In Section 5, we show some numerical experiments which have verified our theoretical results.

\section{Basic Properties and Preliminaries}

Noting that the variable $R$ does not appear in the first two equations of system (4), it is sufficient to consider the following 2-dimensional system.

$$
\left\{\begin{array}{l}
\frac{S_{n+1}-S_{n}}{h}=\Lambda-\mu_{1} S_{n+1}-\frac{\beta S_{n+1} \sum_{i=0}^{\omega} p_{i} I_{n-i}}{1+\alpha S_{n+1}} \\
\frac{I_{n+1}-I_{n}}{h}=\frac{\beta S_{n+1} \sum_{i=0}^{\omega} p_{i} I_{n-i}}{1+\alpha S_{n+1}}-\left(\mu_{2}+\gamma\right) I_{n+1}
\end{array}\right\} n \in(k \tau,(k+1) \tau],
$$

Let $S_{n}=\phi_{n}^{(1)}$ and $I_{n}=\phi_{n}^{(2)}$ for $n=-\omega,-\omega+1,-\omega+2, \cdots, 0$. The initial conditions of the system (5) are given by

$$
\phi_{n}^{(i)} \geq 0(n=-\omega,-\omega+1,-\omega+2, \cdots,-1, i=1,2) \text { and } \phi_{0}^{(i)}>0(i=1,2) .
$$

For the reduced system (5), at first, we show that the solution has positivity for $n>0$, and bounded above for sufficiently large $n$.

Lemma 1. Let $\left(S_{n}, I_{n}\right)$ be a solution of system (5), with the initial conditions (6), then $S_{n}>0$ and $I_{n}>0$ for all $n>0$. And any solution $\left(S_{n}, I_{n}\right)$ of system (5) satisfies $\limsup _{n \rightarrow+\infty}\left(S_{n}+I_{n}\right)<\Lambda / \mu$, where $\mu=\min \left\{\mu_{1}, \mu_{2}+\gamma\right\}$.

Proof. From the initial condition (6) and the first and second equations of system (5), we have

$$
S_{1}=S_{0}+h\left(\Lambda-\mu_{1} S_{1}-\frac{\beta S_{1} \sum_{i=0}^{\omega} p_{i} I_{-i}}{1+\alpha S_{1}}\right)
$$

and

$$
I_{1}=\frac{I_{0}\left(1+\alpha S_{1}\right)+h \beta S_{1} \sum_{i=0}^{\infty} p_{i} I_{-i}}{\left(1+\alpha S_{1}\right)\left(1+h\left(\mu_{2}+\gamma\right)\right)} .
$$

Let $x=S(1)$. It follows from (7) that $x$ satisfies the following equation

$$
\phi(x) \triangleq x-S_{0}-h\left(\Lambda-\mu_{1} x-\frac{\beta x \sum_{i=0}^{\omega} p_{i} I_{-i}}{1+\alpha x}\right)=0 .
$$

Since $\phi(x)$ is monotonically increasing with respect to $x$, and $\phi(0)=-S_{0}-h \Lambda<0, \lim _{x \rightarrow+\infty} \phi(x)=+\infty$. Therefore, there exists a unique $\bar{x}>0$ such that $\phi(\bar{x})=0$. This shows that $S_{1}=\bar{x}>0$.

From (8), we can directly obtain $I_{1}>0$.

From the above discussions, we finally have $S_{1}>0$ and $I_{1}>0$. 
When $n=1$, from model (5) we have

$$
S_{2}=S_{1}+h\left(\Lambda-\mu_{1} S_{2}-\frac{\beta S_{2} \sum_{i=0}^{\omega} p_{i} I_{1-i}}{1+\alpha S_{2}}\right)
$$

and

$$
I_{2}=\frac{I_{0}\left(1+\alpha_{2} S_{2}\right)+h \beta S_{2} \sum_{i=0}^{\omega} p_{i} I_{1-i}}{\left(1+\alpha_{2} S_{2}\right)\left(1+h\left(\mu_{2}+\gamma\right)\right)} .
$$

A similar argument as in the above proof for $S_{1}$ and $I_{1}$, we also can obtain that $S_{2}>0$ and $I_{2}>0$. By using the induction, we can finally obtain that $S_{n}>0$ and $I_{n}>0$ for $n \neq k \tau$. Moreover, $S_{n^{+}}=(1-\theta) S_{n}$, $i_{n^{+}}=i_{n}$ for $n=k \tau$. Therefore we can easily obtain that $S_{n}>0$ and $I_{n}>0$ for all $n>0$.

From the system (5), we have

$$
\left\{\begin{array}{l}
S_{n+1}+I_{n+1}=S_{n}+I_{n}+h\left(\Lambda-\mu_{1} S_{n+1}-\left(\mu_{2}+\gamma\right) I_{n+1}\right), \\
S_{n^{+}}=(1-\theta) S_{n}, \quad I_{n^{+}}=I_{n}, \quad n=k \tau .
\end{array}\right.
$$

Thus

$$
S_{n+1}+I_{n+1} \leq \frac{h \Lambda}{1+h \mu}+\frac{1}{1+h \mu}\left(S_{n}+I_{n}\right) \text {, for all } n>0,
$$

where $\mu=\min \left\{\mu_{1}, \mu_{2}+\gamma\right\}$. Consider the following comparison system

$$
U_{n+1}=\frac{h \Lambda}{1+h \mu}+\frac{1}{1+h \mu} U_{n} .
$$

Obviously, system (9) has a globally asymptotically stable equilibrium $U^{*}=\Lambda / \mu$. Hence, according to the comparison principle of the difference equations, we have that

$$
\limsup _{n \rightarrow \infty}\left(S_{n}+I_{n}\right) \leq \frac{\Lambda}{\mu} .
$$

This shows that $\left(S_{n}, I_{n}\right)$ is also ultimately bounded. This completes the proof.

Lemma 2 [14]. Let us consider the following impulsive difference equations:

$$
\begin{cases}u_{n+1}=a+b u_{n}, & n \in(k \tau,(k+1) \tau], \\ u_{n^{+}}=(1-\theta) u_{n}, & n=k \tau,\end{cases}
$$

where $a>0,0<b<1,0<\theta<1$. Then system (11) has a unique positive periodic solution

$$
\tilde{u}_{n}=\frac{a}{1-b}\left(1-\frac{\theta b^{n-k \tau}}{1-(1-\theta) b^{\tau}}\right), \quad n \in[k \tau,(k+1) \tau),
$$

which is globally asymptotically stable.

Lemma 3. Consider the following equation

$$
x_{n+1}=a_{1} \sum_{i=0}^{\omega} p_{i} x_{n-i}+a_{2} x_{n}
$$

where $0<a_{1}<1,0<a_{2}<1, \omega>0, x_{n} \geq 0$ for $-\omega \leq n<0, x_{0}>0, \sum_{i=0}^{\omega} p_{i}=1$. We have

(i) if $a_{1}+a_{2}<1$, then $\lim _{n \rightarrow \infty} x_{n}=0$;

(ii) if $a_{1}+a_{2}>1$, then $\lim _{n \rightarrow \infty} x_{n}=+\infty$.

Proof. From (12), we have 


$$
\begin{gathered}
x_{1}=a_{1} \sum_{i=0}^{\omega} p_{i} x_{-i}+a_{2} x_{0}>0, \\
x_{2}=a_{1} \sum_{i=0}^{\omega} p_{i} x_{1-i}+a_{2} x_{1}>0, \\
\vdots \\
x_{k}=a_{1} \sum_{i=0}^{\omega} p_{i} x_{k-i}+a_{2} x_{k-1}>0 .
\end{gathered}
$$

It is obvious that $x_{n}>0$ for all $n>0$.

Denote $\underline{x}=\min \left\{x_{0}, x_{1}, x_{2}, \cdots, x_{\omega}\right\}, \bar{x}=\max \left\{x_{0}, x_{1}, x_{2}, \cdots, x_{\omega}\right\}$. From (12), we can also obtain

$$
\begin{gathered}
x_{\omega+1}=a_{1} \sum_{i=0}^{\omega} p_{i} x_{\omega-i}+a_{2} x_{\omega} \leq\left(a_{1}+a_{2}\right) \bar{x}, \\
x_{\omega+2}=a_{1} \sum_{i=0}^{\omega} p_{i} x_{\omega+1-i}+a_{2} x_{\omega+1} \leq\left(a_{1}+a_{2}\right) \bar{x}, \\
\vdots \\
x_{2 \omega}=a_{1} \sum_{i=0}^{\omega} p_{i} x_{2 \omega-1-i}+a_{2} x_{2 \omega-1} \leq\left(a_{1}+a_{2}\right) \bar{x} .
\end{gathered}
$$

Suppose that

$$
x_{k \omega+i} \leq\left(a_{1}+a_{2}\right)^{k} \bar{x} \text { for all } i \in Z^{+}, 0 \leq i<\omega
$$

Then we further have

$$
\begin{aligned}
x_{(k+1) \omega+1}= & a_{1} \sum_{i=0}^{\omega} p_{i} x_{k \omega-i}+a_{2} x_{(k+1) \omega} \leq\left(a_{1}+a_{2}\right)^{k+1} \bar{x}, \\
x_{(k+1) \omega+2}= & a_{1} \sum_{i=0}^{\omega} p_{i} x_{k \omega-i+1}+a_{2} x_{(k+1) \omega+1} \\
\leq & a_{1}\left(a_{1}+a_{2}\right)^{k} \bar{x}+a_{2}\left(a_{1}+a_{2}\right)^{k+1} \bar{x} \leq\left(a_{1}+a_{2}\right)^{k+1} \bar{x}, \\
& \vdots \\
x_{(k+2) \omega}= & a_{1} \sum_{i=0}^{\omega} p_{i} x_{(k+1) \omega-1-i}+a_{2} x_{(k+2) \omega-1} \\
\leq & a_{1}\left(a_{1}+a_{2}\right)^{k} \bar{x}+a_{2}\left(a_{1}+a_{2}\right)^{k+1} \bar{x} \leq\left(a_{1}+a_{2}\right)^{k+1} \bar{x} .
\end{aligned}
$$

By Mathematical induction, we can get for any $n>0$, there exist $k \in Z^{+}, i \in Z^{+}$and $0<i \leq \omega$ such that

$$
x_{n}=x_{k \omega+i} \leq\left(a_{1}+a_{2}\right)^{k} \bar{x}
$$

So, $\lim _{n \rightarrow \infty} x_{n}=0$ if $a_{1}+a_{2}<1$.

By the similar arguments to above steps, we can obtain that for any $n>0$, there exist $k \in Z^{+}, \quad i \in Z^{+}$and $0<i \leq \omega$ such that $x_{n}=x_{k \omega+i} \geq\left(a_{1}+a_{2}\right)^{k} \underline{x}$. and $\lim _{n \rightarrow \infty} x_{n}=+\infty$ if $a_{1}+a_{2}>1$. The Lemma 3 is completed.

\section{Global Attractivity of Infection-Free Periodic Solution}

In this section, we begin to analyze system (5) by first demonstrating the existence of an infection-free periodic solution, in which infectious individuals are entirely absent from the population permanently.

$$
\left\{\begin{array}{lc}
S_{n+1}=\frac{h \Lambda}{1+h \mu_{1}}+\frac{1}{1+h \mu_{1}} S_{n}, & n \in(k \tau,(k+1) \tau], \\
S_{n^{+}}=(1-\theta) S_{n}, & n=k \tau .
\end{array}\right.
$$


By Lemma 2, we know that periodic solution of system (13)

$$
\tilde{S}_{n}=\frac{\Lambda}{\mu_{1}}\left(1-\frac{\theta\left(1+h \mu_{1}\right)^{-(n-k \tau)}}{1-(1-\theta)\left(1+h \mu_{1}\right)^{-\tau}}\right), \quad n \in(k \tau,(k+1) \tau]
$$

which is globally asymptotically stable.

Theorem 4. If $R^{*}<1$, then the infection-free periodic solution $\left(\tilde{S}_{n}, 0\right)$ of system (5) is globally attractive, where

$$
R^{*}=\frac{\beta \bar{S}}{\left(\mu_{2}+\gamma\right)(1+\alpha \bar{S})} \text {, and } \bar{S}=\frac{\Lambda}{\mu_{1}}\left\{1-\frac{\theta\left(1+h \mu_{1}\right)^{-\tau}}{1-(1-\theta)(1+h \mu)^{-\tau}}\right\} \text {. }
$$

Proof. Since $R^{*}<1$, we can choose $\epsilon_{1}>0$ sufficiently small such that

$$
\beta\left(\frac{\bar{S}}{1+\alpha \bar{S}}+\epsilon_{1}\right)<\mu_{2}+\gamma
$$

From the first equation of system (5), we have

$$
S_{n+1}=\frac{h \Lambda+S_{n}}{1+h \mu_{1}+\frac{h \beta \sum_{i=0}^{\omega} p_{i} I_{n-i}}{1+\alpha S_{n+1}}}<\frac{h \Lambda+S_{n}}{1+h \mu_{1}} .
$$

Then we consider the following comparison system with pulses:

$$
\left\{\begin{array}{lc}
x_{n+1}=\frac{h \Lambda}{1+h \mu_{1}}+\frac{1}{1+h \mu_{1}} x_{n}, & n \in(k \tau,(k+1) \tau], \\
x_{n^{+}}=(1-\theta) x_{n}, & n=k \tau .
\end{array}\right.
$$

From Lemma 2, we have that the periodic solution of (16)

$$
\tilde{x}_{n}=\frac{\Lambda}{\mu_{1}}\left\{1-\frac{\theta\left(1+h \mu_{1}\right)^{-(n-k \tau)}}{1-(1-\theta)(1+h \mu)^{-\tau}}\right\}, \quad k \tau<n \leq(k+1) \tau,
$$

is globally asymptotically stable. Let $\left(S_{n}, I_{n}\right)$ be the solution of system (5) with initial value (6) and $S_{0}^{+}=S_{0}^{*}$, and $x_{n}$ be the solution of system (16) with initial value $x_{0}^{+}=S_{0}^{*}$. According to the non-negativity of $S_{n}$ and $x_{n}$, there exists an integer $n_{1} \in Z^{+}$such that

$$
S_{n} \leq x_{n}<\tilde{x}_{n}+\epsilon_{1}, \quad k \tau<n \leq(k+1) \tau, \quad k \geq n_{1},
$$

that is for all $n>n_{1} \tau$,

$$
\begin{aligned}
S_{n} & <\frac{\Lambda}{\mu_{1}}\left\{1-\frac{\theta\left(1+h \mu_{1}\right)^{-(n-k \tau)}}{1-(1-\theta)(1+h \mu)^{-\tau}}\right\}+\epsilon_{1} \\
& \leq \frac{\Lambda}{\mu_{1}}\left\{1-\frac{\theta\left(1+h \mu_{1}\right)^{-\tau}}{1-(1-\theta)(1+h \mu)^{-\tau}}\right\}+\epsilon_{1} \\
& \doteq \bar{S}+\epsilon_{1} .
\end{aligned}
$$

Further, from the second equation of system (5), we have 


$$
\begin{aligned}
I_{n+1} & =\frac{h \beta S_{n+1} \sum_{i=0}^{\omega} p_{i} I_{n-i}}{\left(1+\alpha S_{n+1}\right)\left(1+h \mu_{2}+h \gamma\right)}+\frac{1}{1+h \mu_{2}+h \gamma} I_{n} \\
& \leq \frac{h \beta\left(\bar{S}+\epsilon_{1}\right)}{\left(1+\alpha\left(\bar{S}+\epsilon_{1}\right)\right)\left(1+h \mu_{2}+h \gamma\right)} \sum_{i=0}^{\infty} p_{i} I_{n-i}+\frac{1}{1+h \mu_{2}+h \gamma} I_{n} \\
& \leq \frac{h \beta}{\left(1+h \mu_{2}+h \gamma\right)}\left\{\frac{\bar{S}}{1+\alpha \bar{S}}+\epsilon_{1}\right\} \sum_{i=0}^{\infty} p_{i} I_{n-i}+\frac{1}{1+h \mu_{2}+h \gamma} I_{n}
\end{aligned}
$$

for $n>k \tau, k \geq n_{1}$. Then we consider the following comparison equation:

$$
y_{n+1}=\frac{h \beta}{\left(1+h \mu_{2}+h \gamma\right)}\left\{\frac{\bar{S}}{1+\alpha \bar{S}}+\epsilon_{1}\right\} \sum_{i=0}^{\infty} p_{i} y_{n-i}+\frac{1}{1+h \mu_{2}+h \gamma} y_{n} .
$$

From (15) and Lemma 3, we have $\lim _{n \rightarrow \infty} y_{n}=0$.

Let $y_{n}$ be the solution of (20). We choose a constant value as the initial conditions $I_{i}$ and $y_{i}(i=-\omega,-\omega+1, \cdots, 0)$. By the non-negativity of $I_{n}$ and $\limsup _{n \rightarrow \infty} I_{n} \leq \limsup _{n \rightarrow \infty} y_{n}=0$. Therefore, for any sufficiently small $\epsilon_{2}>0$, there exists an integer $n_{2}>n_{1}$, such that $I_{n}<\epsilon_{2}$ for all $n>n_{2} \tau$. From the first equation of system (5), we have

$$
S_{n+1}>\frac{h \Lambda}{1+\mu_{1}+\beta \epsilon_{2}}+\frac{1}{1+\mu_{1}+\beta \epsilon_{2}} S_{n}, \quad \text { for } n>n_{2} \tau .
$$

Consider the following comparison system with pulse:

$$
\left\{\begin{array}{lc}
z_{n+1}=\frac{h \Lambda}{1+\mu_{1}+\beta \epsilon_{2}}+\frac{1}{1+\mu_{1}+\beta \epsilon_{2}} z_{n}, & n \in(k \tau,(k+1) \tau], \\
z_{n^{+}}=(1-\theta) z_{n}, & n>n_{2},
\end{array}\right.
$$

From Lemma 2, we obtain the globally asymptotically stable periodic solution of (21) $\tilde{z}_{n}$, i.e.

$$
\tilde{z}_{n}=\frac{\Lambda}{\mu_{1}+\beta \epsilon_{2}}\left\{1-\frac{\theta\left(1+h \mu_{1}+h \beta \epsilon_{2}\right)^{-(n-k \tau)}}{1-(1-\theta)\left(1+h \mu+h \beta \epsilon_{2}\right)^{-\tau}}\right\} \text {. }
$$

Let $z_{n}$ be the solution of (21) with initial value (6) and $S_{0}^{+}=S_{0}^{*}$, and $z_{n}$ be the solution of system (16) with initial value $z_{0}^{+}=S_{0}^{*}$. By the non-negativity of $S_{n}$ and $z_{n}$, there exists an integer $n_{3}>n_{2}$ such that

$$
S_{n} \geq z_{n}>\tilde{z}_{n}-\epsilon_{2}, \quad k \tau<n \leq(k+1) \tau, \quad k>n_{3} .
$$

Since $\epsilon_{1}$ and $\epsilon_{2}$ are sufficiently small. From (17) and (22), we know that

$$
\tilde{S}_{n}=\frac{\Lambda}{\mu_{1}}\left\{1-\frac{\theta\left(1+h \mu_{1}\right)^{-(n-k \tau)}}{1-(1-\theta)(1+h \mu)^{-\tau}}\right\}, \quad k \tau<n \leq(k+1) \tau .
$$

is globally attractive.

Hence, the infection-free periodic solution $\left(\tilde{S}_{n}, 0\right)$ of system (5) is globally attractive. The proof is completed.

\section{Permanence}

In this section, we obtain sufficient condition for permanence of system (5). Denote two quantities

$$
R_{*}=\frac{\beta \underline{S}}{\left(\mu_{2}+\gamma\right)(1+\alpha) \underline{S}}
$$

and 


$$
I^{*}=\min \left\{\frac{\mu_{1}}{4 \beta}, \frac{\mu_{1}^{2}\left(\mu_{2}+\gamma\right)\left(R_{*}-1\right)}{8 \Lambda \beta^{2}}\right\},
$$

where $\underline{S}=(1-\theta) \bar{S}$, and $\bar{S}$ is defined in Theorem 4. Obviously, $I^{*}>0$ if $R_{*}>1$.

Theorem 5. Suppose $R_{*}>1$. Then there is a positive constant $q$ such that each solution $\left(S_{n}, I_{n}\right)$ of system (5) satisfies

$$
I_{n} \geq q \text {, for } n \text { large enough. }
$$

Proof. Let $\left(S_{n}, I_{n}\right)$ be any solution of system (5) with initial condition (6). We claim that for any $m_{0}>0$, it is impossible that $I_{n}<I^{*}$ for all $n>m_{0}$. Suppose that the claim is not valid. Then there is a $m_{0}>0$ such that $I_{n}<I^{*}$ for all $n>m_{0}$.

It follows from the first equation of (5), that for $n>m_{0}+\omega$,

$$
S_{n+1}>\frac{h \Lambda+S_{n}}{1+h \mu_{1}+h \beta I^{*}} \text {. }
$$

Consider the following comparison impulsive system for $n>m_{0}+\omega$,

$$
\left\{\begin{array}{l}
u_{n+1}=\frac{h \Lambda}{1+h \mu_{1}+h \beta I^{*}}+\frac{1}{1+h \mu_{1}+h \beta I^{*}} u_{n}, \quad n \in(k \tau,(k+1) \tau], \\
u_{n^{+}}=(1-\theta) u_{n}, \quad n=k \tau .
\end{array}\right.
$$

By Lemma 2, we know that the periodic solution of system (25)

$$
\tilde{u}_{n}=\frac{\Lambda}{\mu_{1}+\beta I^{*}}\left(1-\frac{\theta\left(1+h \mu_{1}+h \beta I^{*}\right)^{-(n-k \tau)}}{1-(1-\theta)\left(1+h \mu_{1}+h \beta I^{*}\right)^{-\tau}}\right), \quad n \in(k \tau,(k+1) \tau],
$$

which is globally asymptotically stable.

From (26), we can get

$$
\begin{aligned}
\tilde{u}_{n} & =\frac{\Lambda}{\mu_{1}+\beta I^{*}}\left(1-\frac{\theta\left(1+h \mu_{1}+h \beta I^{*}\right)^{-(n-k \tau)}}{1-(1-\theta)\left(1+h \mu_{1}+h \beta I^{*}\right)^{-\tau}}\right) \\
& \geq \frac{\Lambda}{\mu_{1}+\beta I^{*}} \frac{(1-\theta)\left(1-\left(1+h \mu_{1}+h \beta I^{*}\right)^{-\tau}\right)}{1-(1-\theta)\left(1+h \mu_{1}+h \beta I^{*}\right)^{-\tau}} \\
& \geq \frac{\Lambda}{\mu_{1}+\beta I^{*}} \frac{(1-\theta)\left(1-\left(1+h \mu_{1}\right)^{-\tau}\right)}{1-(1-\theta)\left(1+h \mu_{1}\right)^{-\tau}}=\frac{\mu_{1}}{\mu_{1}+\beta I^{*}} \underline{S} .
\end{aligned}
$$

Let $\left(S_{n}, I_{n}\right)$ be the solution of system (5) with initial values (6), $u_{n}$ be the solution of system (25) with initial value $u_{0}^{+}=S_{0}^{+}$. By comparison theorem, we know that, for $\varepsilon=\frac{\beta I^{*} \underline{S}}{\mu_{1}+\beta I^{*}}$, there exists $m_{1}\left(>m_{0}+\omega\right)$ such that the following inequality holds for $n>m_{1}$

$$
S_{n}>u_{n}-\varepsilon
$$

It follows from (27) that

$$
S_{n}>u_{n}-\varepsilon \geq \frac{\mu_{1}}{\mu_{1}+\beta I^{*}} \underline{S}-\frac{\beta I^{*} \underline{S}}{\mu_{1}+\beta I^{*}} \geq \frac{\mu_{1}-2 \beta I^{*}}{\mu_{1}} \underline{S}
$$

for $n>m_{1}$. It follows from (23) and (28) that for $n>m_{1}-1$, 


$$
\begin{aligned}
\frac{\beta S_{n+1}}{1+\alpha S_{n+1}}> & \frac{\beta \frac{\mu_{1}-2 \beta I^{*}}{\mu_{1}} \underline{S}}{1+\alpha \frac{\mu_{1}-2 \beta I^{*}}{\mu_{1}} \underline{S}}=\frac{\beta \underline{S}}{1+\alpha \underline{S}+\frac{2 \beta I^{*}}{\mu_{1}-2 \beta I^{*}}} \geq \frac{\beta \underline{S}}{1+\alpha \underline{S}+\frac{4 \beta I^{*}}{\mu_{1}}} \\
& \geq \frac{\beta \underline{S}}{1+\alpha \underline{S}}-\beta \underline{S} \frac{4 \beta I^{*}}{\mu_{1}} \geq\left(\mu_{2}+\gamma\right) R_{*}-\frac{4 \Lambda \beta^{2} I^{*}}{\mu_{1}^{2}} \\
& \geq\left(\mu_{2}+\gamma\right) R_{*}-\frac{1}{2}\left(\mu_{2}+\gamma\right)\left(R_{*}-1\right) \geq \frac{R_{*}+1}{2}\left(\mu_{2}+\gamma\right),
\end{aligned}
$$

Set

$$
I_{l}=\min _{i \in\left[m_{1}, m_{1}+\omega\right]} I_{i} .
$$

We will show that $I_{n} \geq I_{l}$ for all $n \geq m_{1}$. Suppose the contrary. Then there is a $M_{0} \geq 0$ such that $I_{n} \geq I_{l}$ for $m_{1} \leq n \leq m_{1}+\omega+M_{0}$ and $I_{m_{1}+\omega+M_{0}}<I_{l}$.

$$
\begin{aligned}
& I_{m_{1}+\omega+M_{0}}= \frac{h \frac{\beta S_{m_{1}+\omega+M_{0}-1} I_{m_{1}+M_{0}-1}}{1+\alpha S_{m_{1}+\omega+M_{0}-1}}+I_{m_{1}+\omega+M_{0}-1}}{1+h \mu_{2}+h \gamma} \\
& \geq \frac{1+h\left(\frac{R_{*}+1}{2}\left(\mu_{2}+\gamma\right)\right)}{1+h \mu_{2}+h \gamma} I_{l} \geq I_{l} .
\end{aligned}
$$

This is a contradiction. Thus, $I_{n} \geq I_{l}$ for all $n \geq m_{1}$.

Let us consider any positive solution $\left(S_{n}, I_{n}\right)$ of system (5). According to this solution, we define

$$
V_{n}=I_{n}+h W_{n} \text {, }
$$

and

$$
W_{n}=\sum_{j=0}^{\omega}\left\{p_{j}\left(\sum_{k=n-j}^{n} \frac{\beta S_{j+k+1} I_{k}}{1+\alpha S_{j+k+1}}\right)\right\} .
$$

Since

$$
\begin{aligned}
W_{n+1}-W_{n} & =\sum_{j=0}^{\infty}\left\{p_{j}\left(\sum_{k=n+1-j}^{n+1} \frac{\beta S_{j+k+1} I_{k}}{1+\alpha S_{j+k+1}}\right)\right\}-\sum_{j=0}^{\infty}\left\{p_{j}\left(\sum_{k=n-j}^{n} \frac{\beta S_{j+k+1} I_{k}}{1+\alpha S_{j+k+1}}\right)\right\} \\
& =\sum_{j=0}^{\infty}\left\{p_{j}\left(\frac{\beta S_{j+n+2} I_{n+1}}{1+\alpha S_{j+n+2}}-\frac{\beta S_{n+1} I_{n-j}}{1+\alpha S_{n+1}}\right)\right\}
\end{aligned}
$$

It follows from (29), we have that for $n>m_{1}$,

$$
\begin{aligned}
V_{n+1}-V_{n} & =h\left\{\frac{\beta S_{n+1} \sum_{i=0}^{\omega} p_{i} I_{n-i}}{1+\alpha S_{n+1}}-\left(\mu_{2}+\gamma\right) I_{n+1}\right\}+h \sum_{j=0}^{\omega}\left\{p_{j}\left(\frac{\beta S_{j+n+2} I_{n+1}}{1+\alpha S_{j+n+2}}-\frac{\beta S_{n+1} I_{n-j}}{1+\alpha S_{n+1}}\right)\right\} \\
& =h \sum_{j=0}^{\omega}\left\{p_{j} \frac{\beta S_{j+n+2} I_{n+1}}{1+\alpha S_{j+n+2}}\right\}-h\left(\mu_{2}+\gamma\right) I_{n+1} \\
& \geq h \frac{R_{*}+1}{2}\left(\mu_{2}+\gamma\right) I_{n+1}-h\left(\mu_{2}+\gamma\right) I_{n+1} \geq h \frac{R_{*}-1}{2}\left(\mu_{2}+\gamma\right) I_{l},
\end{aligned}
$$


which implies that as $n \rightarrow \infty, V_{n} \rightarrow \infty$. This contradicts $V_{n} \leq \frac{\Lambda}{\mu_{1}}\left(1+h \beta \frac{\Lambda}{\mu_{1}}\right)$. Hence, the claim is proved.

By the claim, we are left to consider two cases.

Case 1. $I_{n} \geq I^{*}$ for all large $n$. The conclusion is evident in this case.

Case 2. $I_{n}$ oscillates about $I^{*}$ for all large $n$. Set $n^{\prime}\left(>m_{1}\right)$ and $\rho \geq 0$ satisfy

$$
I_{n^{\prime}-1} \geq I^{*}, I_{n^{\prime}+\rho+1} \geq I^{*} \text {, and } I_{n}<I^{*} \text { for } n \in\left[n^{\prime}, n^{\prime}+\rho\right] \text {. }
$$

Let $k^{\prime}$ be the smallest integer such that $k^{\prime} \tau$ is strictly exceeding $n^{\prime}$.

Denote

$$
\eta=\max \left\{\left[\frac{\ln \Lambda+\ln \left(\mu_{+} \beta I^{*}\right)-\ln \left(\mu \beta I^{*} \underline{S}\right)}{\tau \ln \left(1+h \mu_{1}\right)}\right]+1,1\right\} .
$$

Subcase 2.1. If $n^{\prime}+\rho \leq\left(k^{\prime}+\eta\right) \tau$, then from the second equation of the system (5), for $n \in\left[n^{\prime}, n^{\prime}+\rho\right]$, we have

$$
\begin{aligned}
I_{n} & \geq \frac{I_{n-1}}{1+h \mu_{2}+h \gamma} \geq \frac{I_{n-2}}{\left(1+h \mu_{2}+h \gamma\right)^{2}} \geq \cdots \geq \frac{I_{n^{\prime}-1}}{\left(1+h \mu_{2}+h \gamma\right)^{n-n^{\prime}+1}} \\
& \geq\left(1+h \mu_{2}+h \gamma\right)^{-(\eta+1) \tau} I^{*} \doteq q
\end{aligned}
$$

Subcase 2.2. If $n^{\prime}+\rho>\left(k^{\prime}+\eta\right) \tau$, we shall consider the following two subcases, respectively.

(a) If $n^{\prime}<n \leq\left(k^{\prime}+\eta\right) \tau$, it follows from (35) that $I_{n} \geq q$;

(b) If $\left(k^{\prime}+\eta\right) \tau<n \leq n^{\prime}+\rho$, we firstly claim that $S_{n} \geq \frac{\mu_{1}-2 \beta I^{*}}{\mu_{1}} \underline{S}$ for $n \geq\left(k^{\prime}+\eta-1\right) \tau$.

From the first equation of comparison system (25) for $k \tau<n \leq(k+1) \tau$, we obtain

$$
u_{n}=\frac{\Lambda}{\mu_{1}+\beta I^{*}}\left(1-\left(1+h \mu_{1}+h \beta I^{*}\right)^{-(n-k \tau)}\right)+\left(1+h \mu_{1}+h \beta I^{*}\right)^{-(n-k \tau)} u_{k \tau},
$$

where $u_{k} \tau$ is the number of $u_{n}$ immediately after the $k$ th pulse vaccination at time $n=k \tau$. Using the second equation of (25), we reduce the stroboscopic map such that

$$
u_{(k+1) \tau^{+}}=(1-\theta) \frac{\Lambda}{\mu_{1}+\beta I^{*}}\left(1-\left(1+h \mu_{1}+h \beta I^{*}\right)^{-\tau}\right)+(1-\theta)\left(1+h \mu_{1}+h \beta I^{*}\right)^{-\tau} u_{k \tau} .
$$

Therefore, by using the stroboscopic map and (27) we can derive for $\eta^{\prime} \geq \eta-1$,

$$
\begin{aligned}
u_{\left(k+\eta^{\prime}\right) \tau^{+}}= & \frac{\Lambda}{\mu_{1}+\beta I^{*}} \frac{(1-\theta)\left(1-\left(1+h \mu_{1}+h \beta I^{*}\right)^{-\tau}\right)}{1-(1-\theta)\left(1+h \mu_{1}+h \beta I^{*}\right)^{-\tau}}\left(1-(1-\theta)^{\eta^{\prime}}\left(1+h \mu_{1}+h \beta I^{*}\right)^{-\eta^{\prime} \tau}\right) \\
& +\left((1-\theta)^{\eta^{\prime}}\left(1+h \mu_{1}+h \beta I^{*}\right)^{-\eta^{\prime} \tau}\right) u_{k \tau^{+}} \\
\geq & \frac{\Lambda}{\mu_{1}+\beta I^{*}} \frac{(1-\theta)\left(1-\left(1+h \mu_{1}+h \beta I^{*}\right)^{-\tau}\right)}{1-(1-\theta)\left(1+h \mu_{1}+h \beta I^{*}\right)^{-\tau}}\left(1-(1-\theta)^{\eta^{\prime}}\left(1+h \mu_{1}+h \beta I^{*}\right)^{-\eta^{\prime} \tau}\right) \\
\geq & \frac{\Lambda}{\mu_{1}+\beta I^{*}} \frac{(1-\theta)\left(1-\left(1+h \mu_{1}+h \beta I^{*}\right)^{-\tau}\right)}{1-(1-\theta)\left(1+h \mu_{1}+h \beta I^{*}\right)^{-\tau}}-\frac{\Lambda}{\mu_{1}}\left(1+h \mu_{1}\right)^{-\eta^{\prime} \tau} \\
\geq & \frac{\mu_{1}}{\mu_{1}+\beta I^{*}} \underline{S}-\frac{\Lambda}{\mu_{1}}\left(1+h \mu_{1}\right)^{-\eta^{\prime} \tau} \geq \frac{\mu_{1}}{\mu_{1}+\beta I^{*}} \underline{S}-\frac{\beta I^{*} \underline{S}}{\mu_{1}+\beta I^{*}} \geq \frac{\mu_{1}-2 \beta I^{*}}{\mu_{1}} \underline{S},
\end{aligned}
$$


and from (36), we also obtain that

$$
\begin{aligned}
u_{\left(k+\eta^{\prime}\right) \tau+k_{1}}= & \frac{\Lambda}{\mu_{1}+\beta I^{*}}\left(1-\left(1+h \mu_{1}+h \beta I^{*}\right)^{-k_{1}}\right)+\left(1+h \mu_{1}+h \beta I^{*}\right)^{-k_{1}} u_{\left(k+\eta^{\prime}\right) \tau^{+}} \\
\geq & \frac{\Lambda}{\mu_{1}+\beta I^{*}} \frac{(1-\theta)\left(1-\left(1+h \mu_{1}+h \beta I^{*}\right)^{-\tau}\right)}{1-(1-\theta)\left(1+h \mu_{1}+h \beta I^{*}\right)^{-\tau}}\left(1-\left(1+h \mu_{1}+h \beta I^{*}\right)^{-k_{1}}\right) \\
& +\left(1+h \mu_{1}+h \beta I^{*}\right)^{-k_{1}} \frac{\Lambda}{\mu_{1}+\beta I^{*}}\left(\frac{(1-\theta)\left(1-\left(1+h \mu_{1}+h \beta I^{*}\right)^{-\tau}\right)}{1-(1-\theta)\left(1+h \mu_{1}+h \beta I^{*}\right)^{-\tau}}-\frac{\Lambda}{\mu_{1}}\left(1+h \mu_{1}\right)^{-\eta^{\prime} \tau}\right) \\
\geq & \frac{\Lambda}{\mu_{1}+\beta I^{*}} \frac{(1-\theta)\left(1-\left(1+h \mu_{1}+h \beta I^{*}\right)^{-\tau}\right)}{1-(1-\theta)\left(1+h \mu_{1}+h \beta I^{*}\right)^{-\tau}}-\frac{\Lambda}{\mu_{1}}\left(1+h \mu_{1}\right)^{-\eta^{\prime} \tau} \geq \frac{\mu_{1}-2 \beta I^{*}}{\mu_{1}} \underline{S} .
\end{aligned}
$$

It follows from (37)and (38) that for $n \geq\left(k^{\prime}+\eta-1\right) \tau$,

$$
S_{n} \geq u_{n} \geq \frac{\mu_{1}-2 \beta I^{*}}{\mu_{1}} \underline{S}
$$

where $\left(S_{n}, I_{n}\right)$ is the solution of system (5) with $S_{\left(k^{\prime}+\eta-1\right) \tau}=s^{*}$, and $u_{n}$ is the solution of comparison system (25) with $u_{\left(k^{\prime}+\eta-1\right) \tau}=s^{*}$. Obviously, (39) implies that (28) holds when $n \geq\left(k^{\prime}+\eta-1\right) \tau$. Then, proceeding exactly as the proof for the above claim, we see that $I_{n} \geq q$ for $\left(k^{\prime}+\eta\right) \tau<n \leq n^{\prime}+\rho$. Since these positive integer $m_{1}$ and $\rho$ are chosen in arbitrary way, we conclude that $I_{n} \geq q$ for all large $n$. This proof is completed.

Theorem 6. System (5) is permanent provided that $R_{*}>1$.

Proof. Denote $\left(S_{n}, I_{n}\right)$ be any solution of system (5) with initial condition (refc1:cond). From the first equation of system (5), we have

$$
S_{n+1}=\frac{h \Lambda+S_{n}}{1+h \mu_{1}+\frac{h \beta \sum_{i=0}^{\tau} p_{i} I_{n-i}}{1+\alpha S_{n+1}}} \geq \frac{h \Lambda+S_{n}}{1+h \mu_{1}+h \beta \Lambda / \mu}
$$

for sufficiently large $n$. Consider the following comparison system:

$$
\left\{\begin{array}{l}
v_{n+1}=\frac{h \Lambda}{1+h \mu_{1}+h \beta \Lambda / \mu}+\frac{1}{1+h \mu_{1}+h \beta \Lambda / \mu} v_{n}, \quad n \in(k \tau,(k+1) \tau], \\
v_{n^{+}}=(1-\theta) v_{n}, \quad n=k \tau .
\end{array}\right.
$$

According to Lemma 2, we know that for any sufficiently small $\varepsilon_{s}$, there exists a sufficiently large $n^{\prime}$ such that

$$
S_{n} \geq v_{n}>\tilde{v}_{n}-\varepsilon_{s} \geq \frac{\Lambda}{m_{1}+\Lambda \beta / \mu}\left\{\frac{(1-\theta)\left(1-(1+h \mu+h \Lambda \beta / \mu)^{-\tau}\right)}{1-(1-\theta)(1+h \mu+h \Lambda \beta / \mu)^{-\tau}}\right\}-\varepsilon_{s} \triangleq m_{s}>0,
$$

for all $n>n^{\prime}$. By Lemma 1 and Theorem 5, we can obtain system (5) is permanent. The proof of Theorem 6 is complete.

\section{Numerical Simulation and Discussion}

We have formulated a discretized SIR epidemic model with pulse vaccination and time delay. We establish some threshold conditions for permanence and extinction of the disease. To illustrate the analytical results, we do some numerical simulations. 
Set $\Lambda=10, \quad \mu_{1}=0.1, \quad \mu_{2}=0.15, \quad \gamma=0.05, \quad \alpha=1, \quad h=1, \quad \omega=2, \tau=8, \quad p_{i}=1 / 3(i=0,1,2)$, then system (5) becomes

$$
\left\{\begin{array}{l}
S_{n+1}-S_{n}=10-0.1 S_{n+1}-\frac{\beta S_{n+1} \sum_{i=0}^{2} \frac{1}{3} I_{n-i}}{1+S_{n+1}}, \\
I_{n+1}-I_{n}=\frac{\beta S_{n+1} \sum_{i=0}^{2} \frac{1}{3} I_{n-i}}{1+S_{n+1}}-(0.15+0.05) I_{n+1}, \\
\begin{array}{l}
S_{n^{+}}=(1-0.25) S_{n}, \\
I_{n^{+}}=I_{n},
\end{array}
\end{array}\right\} n=8 k .
$$

Let $\beta=0.18$, then $R^{*}=0.88916<1$. According to Theorem 4, we know that the disease will die out (see Figure 1). Let $\beta=0.214$, then $R^{*}=1.0529>1$. According to Theorem 5 , we know that the disease will be permanent (see Figure 2).

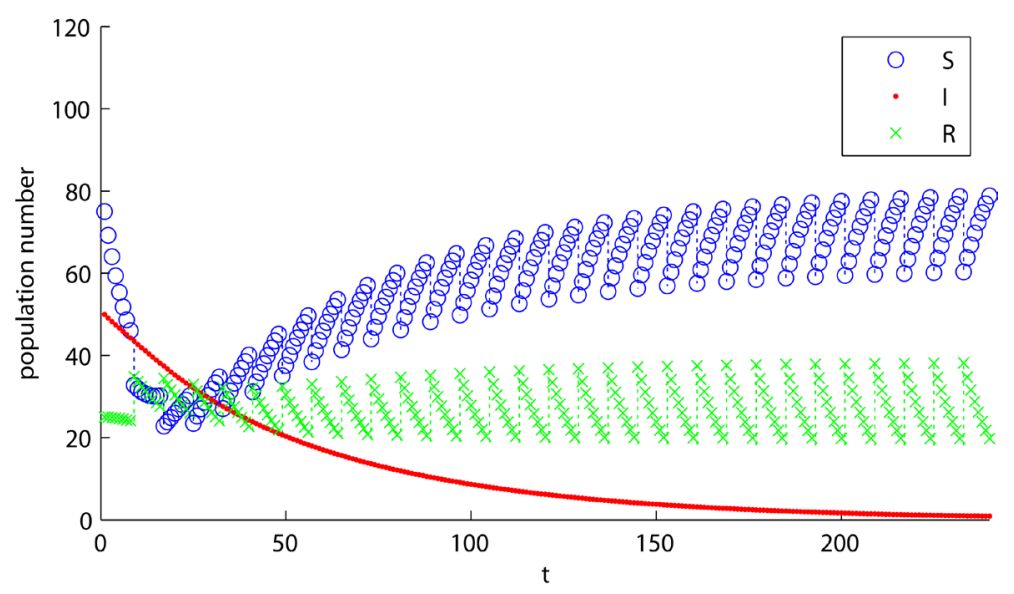

Figure 1. The time series of system (2.1) with initial values are $\phi_{n}^{(1)}=100$, $\phi_{n}^{(2)}=5$, for $n \in[-\omega, 0] . \quad R^{*}=0.88916$. The disease dies out.

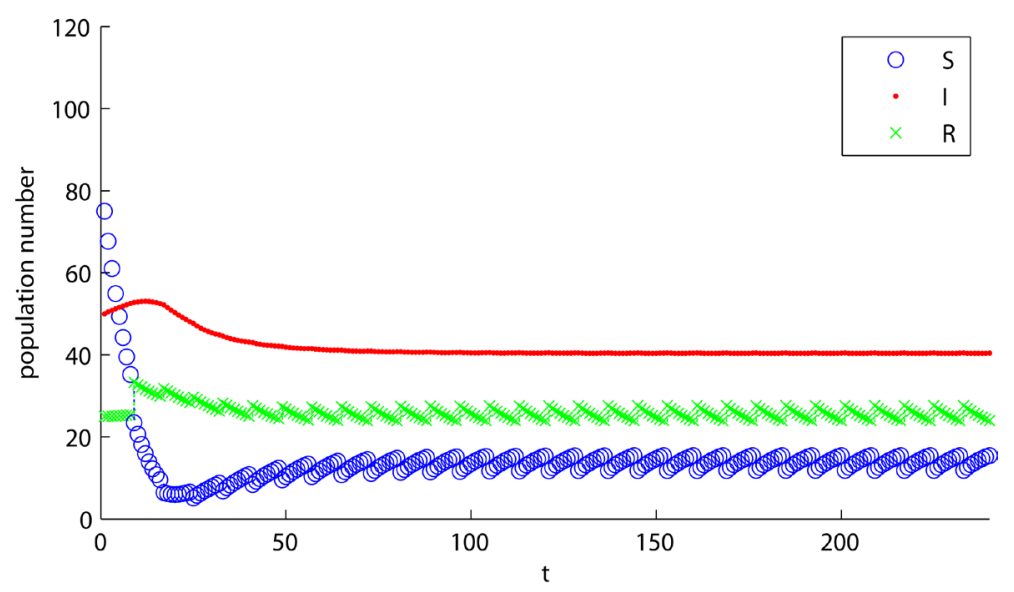

Figure 2. The time series of system (5) with initial values are $\phi_{n}^{(1)}=100$, $\phi_{n}^{(2)}=5$, for $n \in[-\omega, 0] . \quad R_{*}=1.0529$. The disease is permanent. 
Figure 3 shows a bifurcation diagram for stroboscopic map of system (2.1) with the infection rate $\beta$ as the bifurcation parameter. This is illustrated in Figure 4 by the curve $I_{\infty}$ (the number of infection individuals at the equilibrium) that varies with $\beta$. We can observe that the value of $I_{\infty}$ increases as $\beta$ increases, and $I_{\infty}$ is hypersensitive when $\beta \in(0.2,0.215)$, or else it is insensitive.

Figure 4 shows a bifurcation diagram for stroboscopic map system (2.1) with pulse vaccination rate $\theta$ as the bifurcation parameter (for which $R_{*}>1$ ). In this case, numerical result implies that there is unique positive equilibrium of stroboscopic map for all $\theta$, that is, there is a positive periodic solution of system (2.1) for all $\theta$. As Figure 5 and Figure 6 shown, it can be seen that the positive equilibrium is globally attractive.

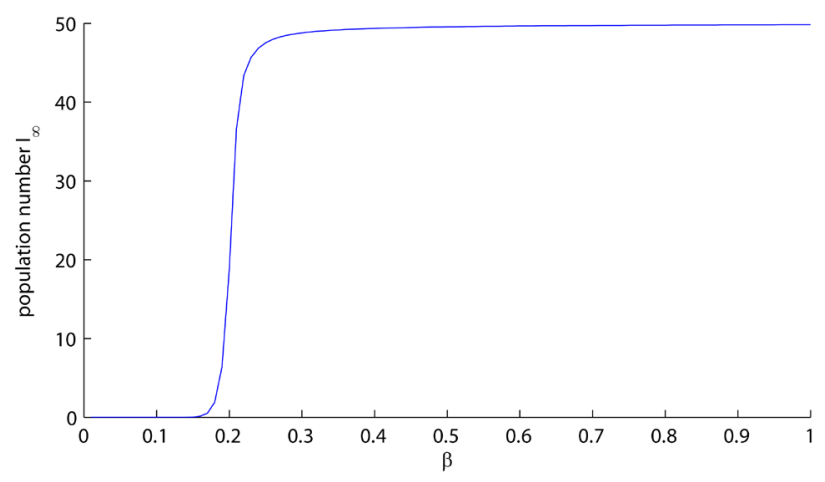

Figure 3. The bifurcation diagram the unique endemic equilibrium (the component $I$ of infectious individuals regarding $\beta$ as the bifurcation parameter, all other parameters are same as in model (5.1)).

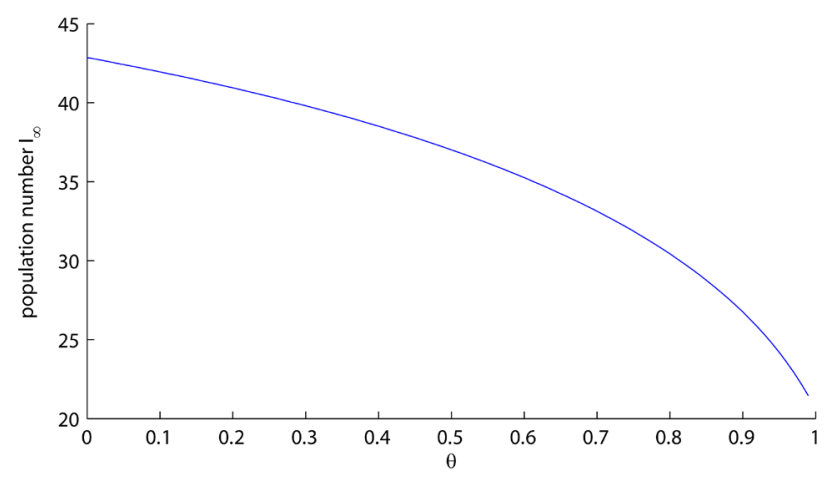

Figure 4. The bifurcation diagram the unique endemic equilibrium (the component $I$ of infectious individuals regarding $\theta$ as the bifurcation parameter, all other parameters are same as in model (5.1) except for $\beta=0.214$ ).

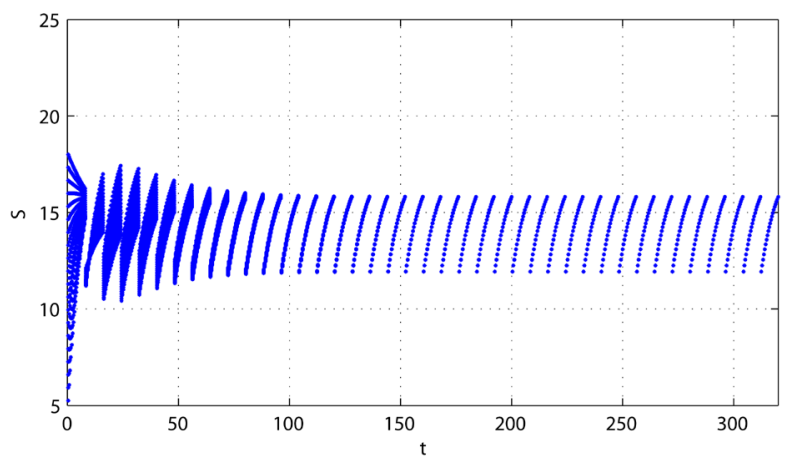

(a)

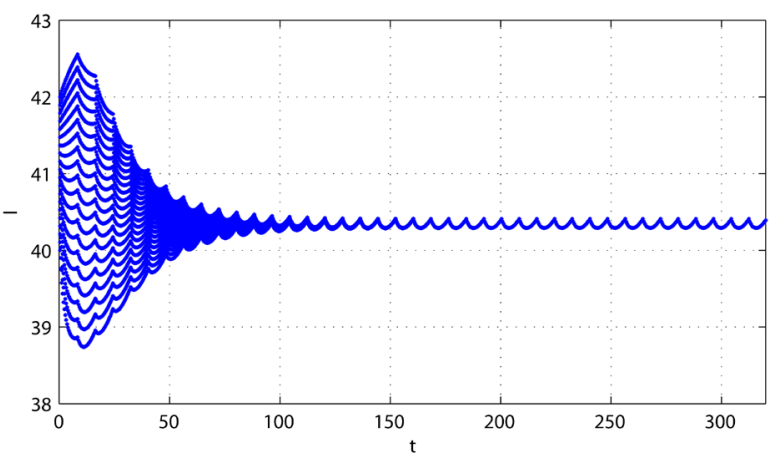

(b)

Figure 5. Time series of system (2.1). $\quad \theta=0.214, R_{*}=1.0529$. 


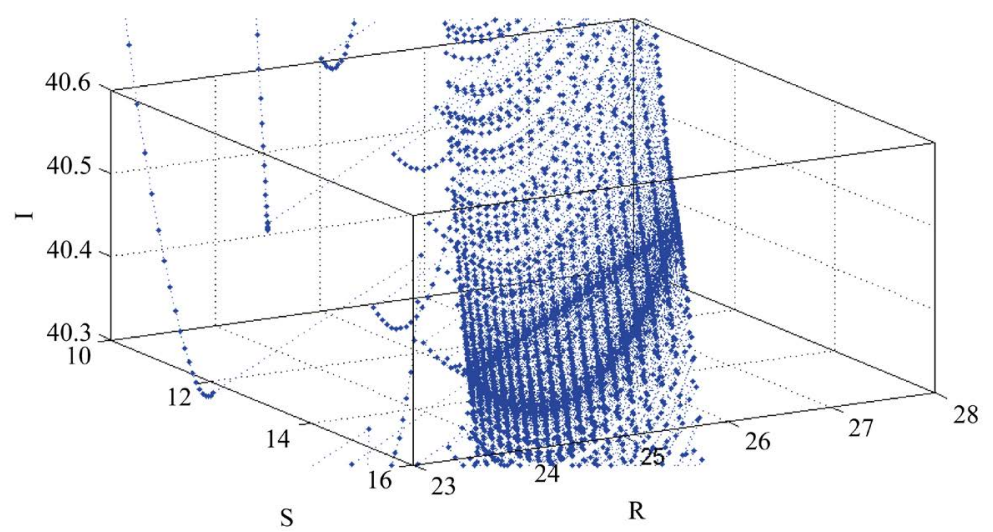

Figure 6. The phase diagram of system (2.1). $R_{*}=1.0529$.

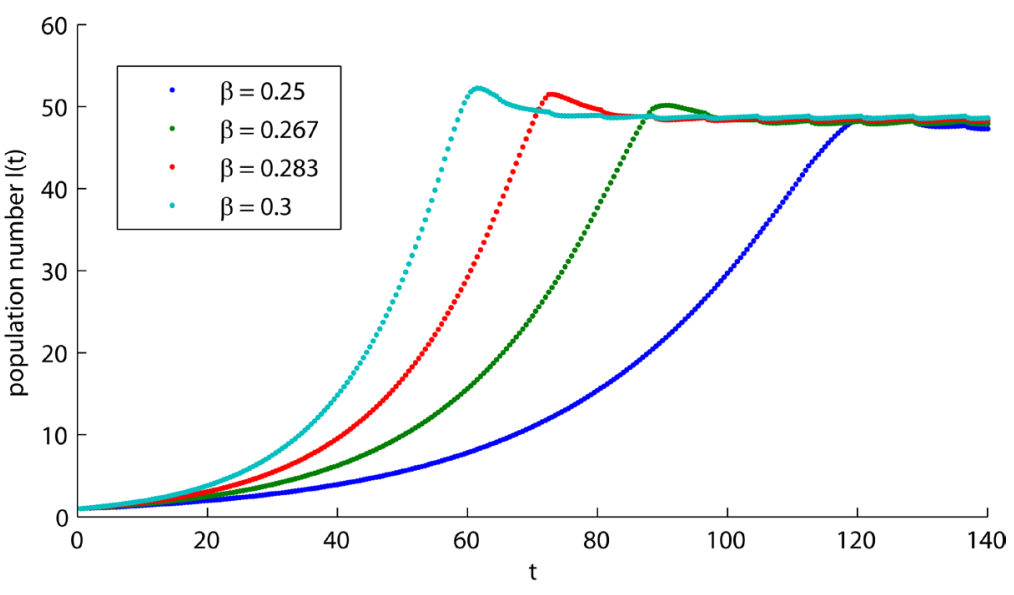

Figure 7. The tendency of the infected individuals $I$ with different values of $\beta$.

Therefore, an interesting open problem is proposed whether we can prove that the positive periodic solution of model (2.1) is globally attractive as $R_{*}>1$.

Finally, the numerical simulations of the stroboscopic map of model on the number of infected individuals with different values of $\beta$ are shown in Figure 7. It shows that the number of infected individuals will increase steadily in next few days, then reach the peak and begin a slow decline, and finally become stable. The greater the value $\beta$, the bigger the peak value and the earlier the peak appears. Our result implies that decreasing infection rate can put off the disease outbreak and reduce the number of infected individuals.

\section{Acknowledgements}

The research has been supported by the Natural Science Foundation of China (11261004, 11561004), the Science and Technology Plan Projects of Jiangxi Provincial Education Department (GJJ14673) and the Social Science Planning Projects of Jiangxi Province (14XW08).

\section{References}

[1] Agur, Z., Cojocaru, L., Mazor, G., et al. (1993) Pulse Mass Measles Vaccination across Age Cohorts. Proceedings of the National Academy of Sciences of the United States of America, 90, 11698-11702. http://dx.doi.org/10.1073/pnas.90.24.11698

[2] Beretta, E. and Takeuchi, Y. (1995) Global Stability of an SIR Epidemic Model with Time Delays. Journal of Mathematical Biology, 33, 250-260. http://dx.doi.org/10.1007/bf00169563

[3] Beretta, E., Hara, T., Ma, W., et al. (2001) Global Asymptotic Stability of an SIR Epidemic Model with Distributed Time Delay. Nonlinear Analysis: Theory, Methods \& Applications, 47, 4107-4115. 
http://dx.doi.org/10.1016/S0362-546X(01)00528-4

[4] Takeuchi, Y., Ma, W. and Beretta, E. (2000) Global Asymptotic Properties of a Delay SIR Epidemic Model with Finite Incubation Times. Nonlinear Analysis: Theory, Methods \& Applications, 42, 931-947. http://dx.doi.org/10.1016/S0362-546X(99)00138-8

[5] Ma, W., Song, M. and Takeuchi, Y. (2004) Global Stability of an SIR Epidemic Model with Time Delay. Applied Mathematics Letters, 17, 1141-1145. http://dx.doi.org/10.1016/j.aml.2003.11.005

[6] Song, M., Ma, W. and Takeuchi, Y. (2007) Permanence of a Delayed SIR Epidemic Model with Density Dependent Birth Rate. Journal of Computational and Applied Mathematics, 201, 389-394. http://dx.doi.org/10.1016/j.cam.2005.12.039

[7] Cooke, K.L. (1979) Stability Analysis for a Vector Disease Model. Rocky Mountain Journal of Mathematics, 9, 31-42. http://dx.doi.org/10.1216/RMJ-1979-9-1-31

[8] Zhang, B. and Liu, Y. (2003) Global Attractivity for Certain Impulsive Delay Differential Equations. Nonlinear Analysis: Theory, Methods \& Applications, 52, 725-736. http://dx.doi.org/10.1016/S0362-546X(02)00129-3

[9] Du, Y. and Xu, R. (2010) A Delayed SIR Epidemic Model with Nonlinear Incidence Rate and Pulse Vaccination. Journal of Applied Mathematics \& Informatics, 1089-1099.

[10] Yan, J., Zhao, A. and Nieto, J.J. (2004) Existence and Global Attractivity of Positive Periodic Solution of Periodic Single-Species Impulsive Lotka-Volterra Systems. Mathematical and Computer Modelling, 40, 509-518. http://dx.doi.org/10.1016/j.mcm.2003.12.011

[11] Zhang, X.B., Huo, H.F., Sun, X.K., et al. (2010) The Differential Susceptibility SIR Epidemic Model with Time Delay and Pulse Vaccination. Journal of Applied Mathematics and Computing, 34, 287-298. http://dx.doi.org/10.1007/s12190-009-0321-y

[12] Capasso, V. and Serio, G. (1978) A Generalization of the Kermack-Mckendrick Deterministic Epidemic Model. Mathematical Biosciences, 42, 43-61. http://dx.doi.org/10.1016/0025-5564(78)90006-8

[13] Lambert, J.D. (1991) Numerical Methods for Ordinary Differential Systems: The Initial Value Problem.

[14] Sekiguchi, M. and Ishiwata, E. (2011) Dynamics of a Discretized SIR Epidemic Model with Pulse Vaccination and Time Delay. Journal of Computational and Applied Mathematics, 236, 997-1008. http://dx.doi.org/10.1016/j.cam.2011.05.040

[15] Mickens, R.E. (1999) Discretizations of Nonlinear Differential Equations Using Explicit Nonstandard Methods. Journal of Computational and Applied Mathematics, 110, 181-185. http://dx.doi.org/10.1016/S0377-0427(99)00233-2

[16] Piyawong, W., Twizell, E.H. and Gumel, A.B. (2003) An Unconditionally Convergent Finite-Difference Scheme for the SIR Model. Applied Mathematics and Computation, 146, 611-625. http://dx.doi.org/10.1016/S0096-3003(02)00607-0

[17] Jódar, L., Villanueva, R.J., Arenas, A.J., et al. (2008) Nonstandard Numerical Methods for a Mathematical Model for Influenza Disease. Mathematics and Computers in Simulation, 79, 622-633. http://dx.doi.org/10.1016/j.matcom.2008.04.008

[18] Erjaee, G.H. and Dannan, F.M. (2004) Stability Analysis of Periodic Solutions to the Nonstandard Discretized Model of the Lotka-Volterra Predator-Prey System. International Journal of Bifurcation and Chaos in Applied Sciences and Engineering, 14, 4301-4308. http://dx.doi.org/10.1142/S0218127404011946

[19] Solis, F.J. and Chen-Charpentier, B. (2004) Nonstandard Discrete Approximations Preserving Stability Properties of Continuous Mathematical Models. Mathematical and Computer Modelling, 40, 481-490. http://dx.doi.org/10.1016/j.mcm.2004.02.028

[20] Su, H. and Ding, X. (2008) Dynamics of a Nonstandard Finite-Difference Scheme for Mackey-Glass System. Journal of Mathematical Analysis and Applications, 344, 932-941. http://dx.doi.org/10.1016/j.jmaa.2008.03.044

[21] Roeger, L.-I.W. (2008) Dynamically Consistent Discrete Lotka-Volterra Competition Models Derived from Nonstandard Finite-Difference Schemes. Discrete and Continuous Dynamical Systems-Series B, 9, 415-429. http://dx.doi.org/10.3934/dcdsb.2008.9.415

[22] Ma, W., Takeuchi, Y., Hara, T., et al. (2002) Permanence of an SIR Epidemic Model with Distributed Time Delays. Tohoku Mathematical Journal, Second Series, 54, 581-591. http://dx.doi.org/10.2748/tmj/1113247650 